\title{
Long-Term Density Evolution through Semi-Analytical and Differential Algebra Techniques
}

Received: date / Accepted: date

\begin{abstract}
This paper introduces and combines for the first time two techniques to allow long-term density propagation in astrodynamics. First, we introduce an efficient method for the propagation of phase space densities based on Differential Algebra (DA) techniques. Second, this DA density propagator is used in combination with a DA implementation of the averaged orbital dynamics through semi-analytical methods. This approach combines the power of orbit averaging with the efficiency of DA techniques.

While the DA-based method for the propagation of densities introduced in this paper is independent of the dynamical system under consideration, the particular combination of DA techniques with averaged equations of motion yields a fast and accurate technique to propagate large clouds of initial conditions and their associated probability density functions very efficiently for long time. This enables the study of the long-term behavior of particles subjected to the given dynamics.

To demonstrate the effectiveness of the proposed approach, the evolution of a cloud of high area-to-mass objects in Medium Earth Orbit is reproduced considering the effects of solar radiation pressure, the Earth's oblateness and luni-solar perturbations. The method can easily propagate 10,000 random

Alexander Wittig

European Space Agency Advanced Concepts Team, ESTEC

Keplerlaan 1, 2200AG Noordwijk, Netherlands

E-mail: alexander.wittig@esa.int

Camilla Colombo

Department of Aerospace Science and Technology, Politecnico di Milano

Via La Masa 34, Milano, 20156, Italy

E-mail: camilla.colombo@polimi.it

Roberto Armellin

Surrey Space Centre, University of Surrey, BA Building, Guildford GU2 7XH, United Kingdom

E-mail: r.armellin@surrey.ac.uk
\end{abstract}


fragments and their density for 1 year within a few seconds on a common desktop PC.

Keywords Density Propagation · Astrodynamics · Semi analytical · Orbit averaging · Differential Algebra

\section{Introduction}

Differential Algebra (DA) is a well established tool for the propagation of clouds of initial conditions in any sufficiently smooth dynamical system (Armellin et al., 2010). It allows the fast and efficient computation of a high order polynomial expansion of the final state as a function of the initial state. As such, it provides a method for the propagation of uncertainties with a single integration (Valli et al., 2013). Besides the huge reduction in computational cost compared to many point-wise integrations, another advantage of DA over conventional point-wise integration is the fact that it yields an analytical expression which approximates the dependence on initial conditions and system parameters.

One of the novel results of this work is that we show how the information contained in the DA flow expansion can be used to not only to propagate points, but also to automatically propagate a probability density function in time at minimal extra computational cost compared to the propagation of just the point cloud. As is the case with the DA propagation method, the DA density propagator is agnostic with respect to the dynamics, i.e. it can be used to propagate densities in arbitrary sufficiently smooth dynamical systems without requiring additional information beyond the differential equations themselves. In particular, it is not necessary to derive and integrate variational equations or analytical solutions to the derivatives of the flow. The result of the DA density propagation is an expansion of the metric tensor used to transform the probability density function with respect to the initial condition. This expansion, once computed, permits the efficient propagation of an arbitrary initial probability density function through the dynamics by mere evaluation of a polynomial as opposed to repeated pointwise integrations.

An area of astrodynamics where the propagation of densities is of particular interest is the long term evolution of orbits around a primary body. In previous research, the continuity equation was used to describe the evolution of the density in time. This approach was successfully applied to describe the population of space debris (McInnes, 1993; Nazarenko, 2003) or the evolution of debris objects after a fragmentation event (Letizia et al., 2015, a), or a cloud of dust particles under the effects of Pointing-Robertson drag (Gor'kavyi, 1997), as well as a swarm of satellites-on-a-chip (SpaceChips) around the Earth (Colombo and McInnes, 2011). In these works the density evolution is described through a partial differential equation, which is then transformed, via the method of characteristics, into a system of ordinary differential equations that describes the dynamics and an adjoint equation for the density, which contains the partial derivatives of the dynamics with respect to the phase space variables (Letizia et al., 2015). The disadvantage 
of the continuity equation method is that it needs to be solved on the domain of initial condition through a grid; therefore, when the problem dimension is high, the derivation of a closed-form expression of the evolution of the density is preferred in order to be competitive with numerical integration and binning methods. This is not always possible and depends on the particular dynamics under consideration.

The effect of orbit perturbations is responsible for the long-term evolution and stability of the motion of natural or artificial satellites in planet-centered dynamics. Solar radiation pressure and planetary oblateness are essential to predict the motion of impact ejecta from Phobos and Deimos in orbits around Mars (Krivov et al., 1995) or high area-to-mass spacecraft around the Earth (Colombo et al., 2012). Similarly, the environment models used for space debris evolution implement the effect of solar radiation pressure, third body perturbations of Sun and Moon, and the effect of the Earth's oblateness (Rossi et al., 1998).

An elegant approach to analyze the effect of orbit perturbations is the semi-analytical averaging technique, which separates the constant, short periodic and long-periodic terms of the disturbing function (Broucke, 2003; Deprit, 1969; G. and P., 1994). The short-term effect of perturbations is eliminated by averaging the variational equations or the corresponding potential over one orbit revolution of the small body. Indeed, averaging corresponds to filtering the higher frequencies of the motion (periodic over one orbit revolution), which typically have small amplitudes. The resulting equations allows a deeper understanding of the dynamics(Colombo, 2015; Colombo et al., 2012), while, from a numerical point of view, the advantage of the averaging approach lies in the reduced computational cost of the dynamics as the stiffness of the problem is reduced, while maintaining sufficient accuracy compatible with typical problem requirements also for long-term integrations.

Semi-analytical and various DA-based techniques were preliminarily compared for long term propagation (Wittig et al., 2014). In particular, a comparison was performed between the direct integration of the full high fidelity equations of motion and the averaged equations for the spacecraft in the Earth's environment implemented in the PlanODyn suite(Colombo, 2015). DA was successfully employed to propagate an initial set of tens of thousands of initial conditions. The combination of DA with semi-analytical techniques demonstrated great potential in reducing the computational time.

In this paper we continue the combination of DA integration techniques with the semi-analytical averaged equations of motion. A DA-implementation of the PlanODyn dynamical model is developed, taking into account solar radiation pressure, the Earth's oblateness and perturbations due to luni-solar gravity (Colombo, 2015). The approach for density propagation presented in this paper is based on the metric tensor for the mapping of probabilities. Its DA-implementation is agnostic to the dynamics and comes at virtually no additional computational cost compared to DA based cloud propagation (Armellin et al., 2010) as it utilizes the information already present in the DA expansion of the flow. It therefore does not require additional derivation or implementation of equations beyond the equations of motion. The proposed method is used to compute the evolution of a cloud of high area-to-mass 


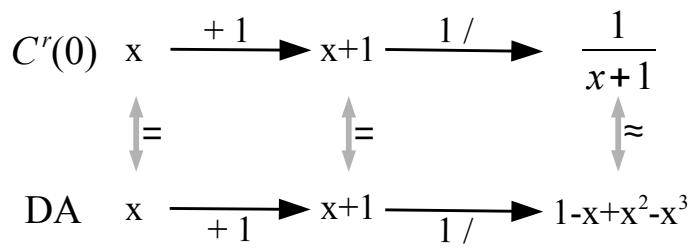

Fig. 1 Evaluation of the expression $1 /(1+x)$ in $C^{r}(0)$ and DA arithmetic.

fragments in Medium Earth Orbit (MEO). In particular, the density evolution is shown in the phase space of eccentricity and longitude with respect to the Sun-Earth direction. This representation provides interesting insight into the underlying characteristics of the dynamics (Colombo et al., 2012). The evolution of the phase space density of high area-to-mass objects is shown for different initial conditions and using different perturbation models for the dynamics.

The article is organized as follows: Section 2 provides a quick overview over the DA technique, while Section 3 introduces the averaged dynamics used in this work. Section 4 introduces the theoretical underpinnings of the density propagation method proposed, while Section 5 focuses on the DA implementation aspects of the density propagation. This is followed by various numerical examples in Section 6. The paper ends with some conclusions and an outlook on future works.

\section{Notes on Differential Algebra}

Differential Algebra techniques allow solving analytical problems through an algebraic approach (Berz, 1999). Similar to the computer representation of real numbers as Floating Point (FP) numbers, DA allows the representation and manipulation of functions on a computer. Each sufficiently often differentiable function $f$ is represented by its Taylor expansion around an expansion point truncated at an arbitrary finite order. Without loss of generality, we choose 0 as the expansion point. Algebraic operations on the space of truncated Taylor polynomials are defined such that they approximate the operations on the function space $C^{r}(0)$ of $r$ times differentiable functions at 0 . More specifically, each operation is defined to result in the truncated Taylor expansion of the correct result computed on the function space $C^{r}(0)$. This yields the so-called Truncated Power Series Algebra (TPSA) (Berz, 1987).

To illustrate the process, consider Figure 1 . The expression $1 /(x+1)$ is evaluated once in $C^{r}(0)$ (top) and then in DA with truncation order 3. Starting with the identity function $x$, we add one to arrive at the function $x+1$, the representation of which is fully accurate in DA as it is a polynomial of order 1 . Continuing the evaluation the multiplicative inversion is performed, resulting in the function $1 /(1+x)$ in $C^{r}(0)$. As this function is not a polynomial any more, it is automatically approximated in DA arithmetic by its truncated Taylor expansion around 0 , given by $1-x+x^{2}-x^{3}$. Note that, by definition of the DA operations, the diagram for each single operation com- 
mutes. That is to say the same result is reached by first Taylor expanding a $C^{r}(0)$ function (moving from the top to the bottom of the diagram) and then performing the DA operation (moving from left to right), or by first performing the $C^{r}(0)$ operation and then Taylor expanding the result.

In addition to algebraic operations, the DA framework can be endowed with natural differentiation and integration operators, completing the structure of a differential algebra. Intrinsic functions, such as trigonometric and exponential functions, are built from elementary algebraic operations (Berz, 1999). This way, Taylor expansions of arbitrary sufficiently smooth functions given by some closed-form expression can be computed fully algebraically in a computer environment.

An implementation of such DA computer routines is available in the software COSY INFINITY (Berz and Makino, 2006) by M. Berz and K. Makino, which is used in to implement the algorithms presented in this paper.

An important application of DA in engineering applications is the expansion of the flow $\varphi\left(t ; x_{0}\right)$ of an Ordinary Differential Equation (ODE) to arbitrary order with respect to initial conditions, integration times and system parameters. The following is a short summary of the underlying concept. For a more complete introduction to DA, as well as a fully worked out illustrative example of a DA based ODE integrator using a simple Euler step, see Valli et al. (2013).

Consider the initial value problem

$$
\left\{\begin{array}{l}
\dot{x}=f(x, t) \\
x\left(t_{0}\right)=x_{0}
\end{array}\right.
$$

and its associated flow $\varphi\left(t ; x_{0}\right)$. By means of classical numerical integration schemes, such as Runge-Kutta or multi-step methods, it is possible to compute the orbit of a single initial condition $x_{0}$ using floating point arithmetic on a computer. Starting instead from the DA representation of an initial condition $x_{0}$, and performing all operations in the numerical integration scheme in DA arithmetic, DA allows propagating the Taylor expansion of the flow around $x_{0}$ forward in time, up to the desired final time $t_{f}$, yielding a polynomial expansion of $\varphi\left(t_{f} ; x_{0}+\delta x_{0}\right)$ up to arbitrary order.

The conversion of standard explicit integration schemes to their DA counterparts is rather straightforward. One simply replaces all operations performed during the execution of the scheme by the corresponding DA operations. Step size control and error estimates are performed only on the constant part of the polynomial, i.e. the reference trajectory of the expansion point. The result is an automatic Taylor expansion of the result of the numerical method (i.e. the numerical approximation to the flow) with respect to any quantity that was initially set to a DA value.

The main advantage of the DA-based approach is that there is no need to derive, implement and integrate variational equations in order to obtain high-order expansions of the flow. As this is achieved by merely replacing algebraic operations on floating-point numbers by DA operations, the method is inherently ODE independent. Furthermore, the efficient implementation of DA e.g. in COSY INFINITY allows us to obtain high-order expansions with limited computational time. 
For the computations in this paper, we use a $7 / 8$ order Runge-KuttaFehlberg integrator scheme with automatic step size control for the integration of the dynamics starting with a DA representation of the initial condition. This enables us to compute the flow expansion to arbitrary order in terms of the initial conditions at final time $t$. While straightforward to implement, the DA integration method can be very time consuming if the right hand side of the ODE is stiff. In particular, in the case of the long term evolution in perturbed 2-body dynamics the fast near-Keplerian motion requires small step sizes. This is not specific to DA but a property found in numerical ODE integration in general. This observation gives rise to the averaging technique described in the next section, which mitigates the impact of this problem by removing the fast motion and replacing it by a smooth mean motion.

\section{Averaged Dynamics}

The orbit evolution in the Earth environment can be expressed in terms of variation of orbital parameters through variational equations in Gauss' form or Lagrange's form (Battin, 1999). When the long-term propagation of the dynamics is required, it is convenient to separate the disturbing function (or disturbing force) in terms of its constant component, short period variations and long period variations. In particular, it is possible to isolate the secular and the long-period effects on the dynamics by eliminating the short period term through the averaging technique. The most common form is obtained by averaging the perturbation over one orbit revolution. Considering that the evolution of the semi-major axis $a$, eccentricity $e$, inclination $i$, anomaly of the ascending node $\Omega$ and anomaly of the pericenter $\omega$ are much slower than the true anomaly $\nu$ or mean anomaly $M$, the variational equations can be averaged over one revolution (i.e. from 0 to $2 \pi$ ) of $M$ considering the other orbital elements as constant. The averaged dynamics can then be numerically integrated to account for the secular and long-term variation of the orbital elements.

In this article we consider the effects of some of the main perturbations for Earth-centered orbits including solar radiation pressure, the effect of the Earth's oblateness and luni-solar perturbations. The expression for the averaged potential to describe the long-term and secular variation of the orbital elements were derived in (Colombo et al., 2012) and (Colombo, 2015), we summarize the key points here for clarity.

The secular and long-period rate of change of the orbital elements due to solar radiation pressure (SRP) and $J_{2}$ are given for example in (Krivov et al., 1995) and were implemented in PlanODyn (Colombo et al., 2012) as:

$$
\begin{aligned}
{\frac{d \bar{\Omega}}{d t} J_{2}}=-\frac{3}{2} J_{2}\left(\frac{R_{E}}{a}\right)^{2} \frac{n}{\left(1-e^{2}\right)^{2}} \cos i \\
{\frac{d \bar{\omega}}{d t} J_{2}}^{2}=\frac{3}{4} J_{2}\left(\frac{R_{E}}{a}\right)^{2} \frac{n}{\left(1-e^{2}\right)^{2}}\left(5 \cos ^{2} i-1\right)
\end{aligned}
$$


for the Earth oblateness, where $J_{2}=1.083 \cdot 10^{-3}, R_{E}$ is the radius of the Earth and $n$ the orbital mean motion, and

$$
\begin{aligned}
\frac{d \bar{e}}{d t}_{S R P}= & \frac{3}{2} \frac{n a_{S R P} a^{2}}{\mu} \sqrt{1-e^{2}} \sum_{k=1}^{6} A_{k} \sin \alpha_{k} \\
\overline{d \bar{i}}_{S R P}= & \frac{3}{2} \frac{n a_{S R P} a^{2} e}{\mu \sqrt{1-e^{2}}} \cos \omega \sum_{k=7}^{9} A_{k} \sin \alpha_{k} \\
\frac{d \bar{\Omega}}{d t}_{S R P}= & \frac{3}{2} \frac{n a_{S R P} a^{2} e}{\mu \sin i \sqrt{1-e^{2}}} \sin \omega \sum_{k=1}^{6} A_{k} \sin \alpha_{k} \\
\frac{d \bar{\omega}}{d t}_{S R P}= & -\cos i \frac{d \bar{\Omega}}{d t} S R P \\
& \frac{3}{2} \frac{n a_{S R P} a^{2}}{\mu} \frac{\sqrt{1-e^{2}}}{e} \sum_{k=1}^{6} A_{k} \sin \alpha_{k}
\end{aligned}
$$

The coefficients $A_{1}$ to $A_{9}$ and the angles $\alpha_{1}$ to $\alpha_{9}$ are function of the orbit's plane orientation $i, \Omega, \omega$, and the longitude of the Sun on the ecliptic $\lambda_{\text {Sun }}$ as well as the obliquity angle $\varepsilon$ of the ecliptic over the equator. $a_{S R P}$ is the characteristic acceleration due to SRP:

$$
a_{S R P}=\frac{p_{S R} c_{R} A}{m}
$$

where $p_{S R}$ is the solar pressure $p_{S R}=4.56 \cdot 10^{-6} \mathrm{~N} / \mathrm{m}^{2}, c_{R}$ the reflectivity coefficient, and $A / m$ the area-to-mass ratio of the spacecraft cross section area $A$ exposed to the Sun. The effect of eclipses is neglected for the moment. The secular variation of semi-major axis due to Earth's oblateness and solar radiation pressure without considering eclipses is zero. The effect of the third body perturbation due to the Moon and the Sun is also considered, by averaging the third body potential written in orbital elements. The expression of the resulting equations can be found in (Colombo, 2015).

For the present work the averaged dynamics algorithm in PlanODyn was implemented in COSY INFINITY. The COSY implementation was successfully validated against the original PlanODyn implementation of both the averaged dynamics and the high-fidelity dynamics. For the high fidelity dynamics the expression of the acceleration due to Solar radiation pressure, Earth's oblateness and luni-solar perturbation were inserted into the Gauss's form of planetary equations (Battin, 1999) and numerically integrated directly.

\section{Density Propagation}

The term density may refer to both a physical density as well as a probability density function (pdf) in the phase space of a given dynamical system. The physical density $N(\boldsymbol{x})$ gives the number of particles in the system that 
occupy an infinitely small phase space volume around the state $\boldsymbol{x}$. The probability density function $p(\boldsymbol{x})$, on the other hand, provides the probability of a particle occupying an infinitely small phase space volume around the state $\boldsymbol{x}$. Mathematically, both formulations are in fact identical except for normalization. The pdf is normalized such that the total integrated probability of a particle occupying any state is 1 ,

$$
\int_{V} p(\boldsymbol{x}) d V=1
$$

while the physical density is normalized such that the integral yields the total number of particles in the system:

$$
\int_{V} N(\boldsymbol{x}) d V=N_{t o t} .
$$

Without loss of generality we shall refer to the density as a pdf for the remainder of this paper.

It is important to note that our notion of density in phase space differs from the notion of spatial density. The spatial density is the density of particles in physical space only, neglecting different velocities present in phase space. In order to pass from the phase space density to the spacial density, it is necessary to integrate the distribution over the velocity.

In general, as a dynamical system evolves with time, the pdf also changes. Thus, the pdf is time dependent: $p_{t}(\boldsymbol{x})$. The goal of this section is to obtain a transformation from the known pdf $p_{t_{0}}(\boldsymbol{x})$ at time $t_{0}$ to the general pdf $p_{t}(\boldsymbol{x})$ at any time $t$. The evolution of the density in a dynamical system where particles are conserved locally, i.e. a system without sources or sinks of particles, is fully described by the local evolution of phase space volume under the effect of the ordinary differential equation governing the motion.

Let the evolution of a dynamical system be described by the first order ordinary differential equation

$$
\frac{d \boldsymbol{x}}{d t}=f(\boldsymbol{x}, t)
$$

where $f: \mathbb{R}^{n} \rightarrow \mathbb{R}^{n}$ is a $C^{1}$ function, i.e. at least once continuously differentiable, and $\boldsymbol{x}(t) \in \mathbb{R}^{n}$ is the state vector of the system. For any given initial condition $\boldsymbol{x}_{0}$ at time $t_{0}$, this ODE has a unique solution $\boldsymbol{x}(t)$ such that $\boldsymbol{x}\left(t_{0}\right)=x_{0}$ and $\boldsymbol{x}(t)$ satisfies Eq. (5).

We define $\varphi_{t}(\boldsymbol{x})$ to be the general solution of the ODE defined by Eq. (5) satisfying

$$
\left\{\begin{array}{l}
\varphi_{t_{0}}(\boldsymbol{x})=\boldsymbol{x} \\
\frac{d \varphi_{t}(\boldsymbol{x})}{d t}=f\left(\varphi_{t}(\boldsymbol{x}), t\right),
\end{array}\right.
$$

i.e. $\varphi_{t}(\boldsymbol{x})$ is the flow of the ODE.

Fixing the time $t, \boldsymbol{y}=\varphi_{t}(\boldsymbol{x})$ defines a map from any initial point $\boldsymbol{x}$ in phase space at time $t_{0}$ to the corresponding final point $\boldsymbol{y}$ in phase space at time $t$. Due to the uniqueness of solutions of the ODE, this map is a 1-to1 , and hence invertible, $C^{1}$ transformation of phase space. We denote the 


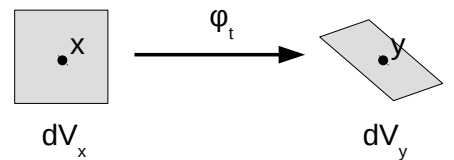

Fig. 2 Illustration of the transformation of an infinitesimal volume $d V_{x}$ to $d V_{y}$ under the map $\varphi_{t}$.

inverse function by $\boldsymbol{x}=\varphi_{t}^{-1}(\boldsymbol{y})$ and the Jacobian with respect to the phase space variables $\boldsymbol{x}$ and $\boldsymbol{y}$ as $J \varphi_{t}(\boldsymbol{x})$ and $J \varphi_{t}^{-1}(\boldsymbol{y})$ respectively.

The problem of obtaining the relationship between $p_{t}(\boldsymbol{x})$ and $p_{t_{0}}(\boldsymbol{x})$ now is reduced to finding the pdf of the dependent variable $\boldsymbol{y}=\varphi_{t}(\boldsymbol{x})$ given the pdf of $\boldsymbol{x}$. This relation between the pdf of a dependent and independent variable is well known in statistics (Soong, 2004, p. 149) and given by

$$
p_{t}(\boldsymbol{y})=p_{t_{0}}\left(\varphi_{t}^{-1}(\boldsymbol{y})\right) \cdot \operatorname{det}\left(J \varphi_{t}^{-1}(\boldsymbol{y})\right),
$$

or written in terms of $\boldsymbol{x}$

$$
p_{t}\left(\varphi_{t}(\boldsymbol{x})\right)=p_{t_{0}}(\boldsymbol{x}) / \operatorname{det}\left(J \varphi_{t}(\boldsymbol{x})\right) .
$$

In Eq. (7) we made use of the fact that $J \varphi_{t}^{-1}(\boldsymbol{y})=\left(J \varphi_{t}(\boldsymbol{x})\right)^{-1}$ and hence

$$
\operatorname{det}\left(J \varphi_{t}^{-1}(\boldsymbol{y})\right)=1 / \operatorname{det}\left(J \varphi_{t}(\boldsymbol{x})\right) .
$$

Eqs. (6) and (7) can be understood intuitively by considering the evolution of infinitesimally small phase space volumes. To derive Eq. (7), consider the infinitesimally small phase space volume $d V_{x}$ centered at $\boldsymbol{x}$. Mapping this infinitesimally small phase space volume $d V_{x}$ forward through the map $\varphi_{t}$ yields another infinitesimally small phase space volume $d V_{y}$ centered at $y=\varphi_{t}(\boldsymbol{x})$ (see Figure 2).

The probability of a particle occupying the phase space volume $d V_{x}$ at time $t_{0}$ is given by $p_{t_{0}}(\boldsymbol{x}) \cdot d V_{x}$, while the probability of a particle occupying $d V_{y}$ at time $t$ is given by $p_{t}(\boldsymbol{y}) \cdot d V_{y}=p_{t}\left(\varphi_{t}(\boldsymbol{x})\right) \cdot d V_{y}$. Due to the principle of probability conservation, both probabilities must be the same, yielding

$$
p_{t_{0}}(\boldsymbol{x}) \cdot d V_{x}=p_{t}\left(\varphi_{t}(\boldsymbol{x})\right) \cdot d V_{y} .
$$

Solving for $p_{t}\left(\varphi_{t}(\boldsymbol{x})\right)$ we obtain

$$
p_{t}\left(\varphi_{t}(\boldsymbol{x})\right)=p_{t_{0}}(\boldsymbol{x}) \cdot d V_{x} / d V_{y} .
$$

Drawing on a result from differential geometry, we recall that the relation between the initial volume $d V_{x}$ and the final volume $d V_{y}$ is given by

$$
d V_{y}=\operatorname{det}\left(J \varphi_{t}(\boldsymbol{x})\right) \cdot d V_{x},
$$

where in differential geometry the quantity inside the determinant is also known as the Riemann or metric tensor (Postnikov, 2001, p. 159). Inserting Eq. (9) into Eq. (8) we obtain Eq. (7). 


\section{DA Implementation}

As the result of the previous section, we obtained the relationship between the densities at different times in a dynamical system given by Eq. (7). In order to compute the new pdf $p_{t}(\varphi(\boldsymbol{x}))$, this equation requires the computation of the determinant of the metric tensor $\operatorname{det}\left(J \varphi_{t}(\boldsymbol{x})\right)$. This in turn requires knowledge of the derivatives of the flow to obtain the Jacobian $J \varphi_{t}(\boldsymbol{x})$.

While it is possible to manually derive the variational equations corresponding to the particular given dynamics and integrate the combined equations of motion in order to obtain the Jacobian $J \varphi_{t}(\boldsymbol{x})$ at the given point, this process has several drawbacks. Firstly, it requires the manual derivation of the variational equations for each and every different ODE. Given the complexity of most realistic dynamical models, this task can become quite tedious and yield very long, difficult, and error-prone expressions. Furthermore, even for a simple 6 dimensional phase space, the dimension of the ODE including the variational equations is already 42 , resulting in significant additional computational effort. Lastly, this procedure only provides the Jacobian and hence the new density at a single point. In order to evaluate the density at many different points, e.g. in a cloud of initial conditions, a separate integration is required for every single evaluation.

We instead use DA to compute an expansion of the new density as given in Eq. (7) without the need of manually calculating analytical flow derivatives or deriving and integrating variational equations. We achieve this by making use of the differential structure of DA. First, a high order flow expansion between times $t_{0}$ and $t$ is computed as described in section 2. Since the final result of this process is a polynomial expansion of $\varphi_{t}(\boldsymbol{x})$ in terms of $\boldsymbol{x}$, it is easy to take the derivative of the polynomial expansion to obtain a polynomial expansion of the Jacobian $J \varphi_{t}(\boldsymbol{x})$.

Performing all the operations in the computation of the metric tensor in DA arithmetic, followed by the DA evaluation of the determinant itself, one readily obtains an expansion of the determinant of the metric tensor. This then allows the fast and efficient evaluation of the density $p_{t}\left(\varphi_{t}(\boldsymbol{x})\right)$ by Eq. (7). As the determinant itself is a polynomial expansion valid in an entire neighborhood of the chosen expansion point, it is possible to evaluate the density $p_{t}\left(\varphi_{t}(\boldsymbol{x})\right)$ not just at the expansion point itself, but at any number of points in its neighborhood without the need for additional integrations by the simple evaluation of a polynomial.

Note that we do not compute the full Taylor expansion of the final pdf $p_{t}\left(\varphi_{t}(\boldsymbol{x})\right)$. Instead, we leave the explicit dependence on the initial pdf $p_{t_{0}}(\boldsymbol{x})$ as per Eq. (7) intact. This has several advantages. Firstly, it is possible to propagate a different pdf without reintegrating the dynamics to recompute the metric tensor. Secondly, expanding the entire pdf directly will typically result in an expansion with poor convergence as the pdf has an asymptotic behavior approaching zero, while all non-constant polynomials of course diverge to $\pm \infty$. Thus a single polynomial expansion will only describe the pdf well in a small, local neighborhood, which for typical pdfs (such as Gaussian distributions) is much smaller than the radius of convergence of the determinant. 
We further remark that the above method is applicable also to the computation of the density as given in Eq. (6). The only difference is that in this case the numerical integration is to be carried out backwards from time $t$ to time $t_{0}$, starting with a DA initial condition $[\boldsymbol{y}]$ at time $t$ to obtain the DA expansion of the expression $\left[\varphi_{t}^{-1}\right](\boldsymbol{y})$.

Which of the two formulations, Eq. (6) or (7), is the better choice is largely problem dependent. Either representation of the pdf has advantages depending on the final goal that is to be achieved with the pdf. In the following section, we compute the density according to Eq. (7), expanding around the area of interest in the prescribed initial density distribution $p_{t_{0}}(\boldsymbol{x})$. For visualizing the final pdf, this formulation is ideal as it at the same time shows the evolution of the initial set in phase space over time, while in addition providing the density at each point in the propagated set.

\section{Numerical Examples}

To demonstrate the proposed method of DA-based propagation of the phase space density in semi-analytical dynamics, we consider an initial condition on a Medium Earth Orbit characterized by the values given in Tab. 1. Objects with high area-to-mass ratio $A / m$ equal to $25 \mathrm{~m}^{2} / \mathrm{kg}$ are considered. The set of initial conditions used in the following propagations is centered around the reference values, with the size in each component as given. As can be seen, in this study we only consider two dimensional statistics in $e$ and $\omega$, while the other elements are considered exact. This is not a restriction of the method but merely serves to simplify the representation of the resulting probability density function in phase space.

In the following, to increase the clarity of the explanation of the cloud evolution, a dynamical model with increasing complexity is used. First, a simplified Sun model is adopted as in Krivov et al. (1995) and Colombo et al. (2012): the ecliptic plane is assumed to be equivalent to the equatorial plane (i.e. the obliquity angle $\varepsilon=0$ ) and the Earth is assumed on a circular orbit around the Sun. The only perturbations considered are solar radiation pressure and $J_{2}$. This will reveal some interesting characteristics of the phase space map as first noted in Colombo and McInnes (2011). Then the model complexity is increased by considering the real ephemerides of the Sun as well as including in the model luni-solar perturbations using a second-degree model of the disturbing potential.

\begin{tabular}{l|r|l}
\hline \hline Element & Reference & Size \\
\hline$a_{0}$ & $23222 \mathrm{~km}$ & $\pm 0 \mathrm{~km}$ \\
$e_{0}$ & 0.08 & \pm 0.03 \\
$i_{0}$ & $0.01 \mathrm{deg}$ & $\pm 0 \mathrm{deg}$ \\
$\Omega_{0}$ & $0 \mathrm{deg}$ & $\pm 0 \mathrm{deg}$ \\
$\omega_{0}$ & $0 \mathrm{deg}$ & $\pm 5.7 \mathrm{deg}$ \\
$M_{0}$ & $0 \mathrm{deg}$ & $\pm 0 \mathrm{deg}$ \\
\hline \hline
\end{tabular}

Table 1 Initial conditions used for the propagations of objects with an area-tomass ratio of $25 \mathrm{~m}^{2} / \mathrm{kg}$. The size refers to a box of uniform density. 


\section{$6.1 J_{2}$ and simplified SRP Dynamics}

The two-body dynamics of a spacecraft with high area-to-mass ratio orbiting the Earth is strongly perturbed by the term of the gravitational field due to the Earth's oblateness and by the effect of solar radiation pressure in the case of large area-to-mass ratio.

The orbit evolution shows interesting behavior in the phase space of eccentricity $e$ and $\phi$, where

$$
\phi=\Omega+\omega-\left(\lambda_{S u n}-\pi\right)
$$

describes the orientation of the orbit perigee with respect to the Sun. As was analyzed in Colombo et al. (2012), if the obliquity angle is assumed to be zero, the system allows some equilibrium solutions which correspond to frozen orbits with respect to the Sun. The equilibrium solution $\phi=0$, existing at semi-major axis below approximately $15000 \mathrm{~km}$ corresponds to a family of heliotropic orbits that maintain their perigee in the direction of the Sun, while $\phi=\pi$, existing for semi-major axis above $13000 \mathrm{~km}$ approximately, corresponds to families of anti-heliotropic orbits, with the apogee frozen in the Sun direction.

Initial conditions around the equilibrium orbit will librate in the phase space of $e-\phi$ around the equilibrium. The eccentricity value of the equilibrium solution depends on the semi-major axis $a$ and the value of the area-tomass ratio $A / m$ of the spacecraft, which can be used as a control parameter to design frozen orbits with respect to the Sun. A detailed description of the possible solutions is given in Colombo et al. (2012).

In this simplified model we study the evolution of the density under a simplified solar ephemeris model and the effect of $J_{2}$ and solar radiation pressure only. As mentioned, the equator is assumed to be on the ecliptic, $\varepsilon=0$ and a circular orbit for the Earth around the Sun is used. Instead of studying the motion in full six dimensional phase space, we focus on the projection into the $e-\phi$ plane.

Figure 3 shows the evolution of an initial set of phase space with initially uniform density (bottom). As the system evolves, the set moves counter clockwise on a periodic orbit. The period of the motion is about 378.3 days. Note that the period of the motion, i.e., one librational loop around the equilibrium point (which corresponds to an anti-heliotropic orbit), should not be confused with the orbit period. Unless otherwise stated we will refer here to the librational loop period. As can be seen, the phase space is compressed during the evolution. However, the compression is not uniform over the initial set, causing the density to become significantly non-uniform over the initial set. After completing one full period, the set returns almost to the initial set and also the density almost returns to the initial uniform distribution.

Figure 4(a) shows the evolution of the minimum and maximum densities at various times during one single loop period. Starting from the uniform distribution of (non-normalized) density 1, both the minimum and the maximum density increases, but after about half a period the difference between the minimum and maximum density becomes largest. On the second half pe- 


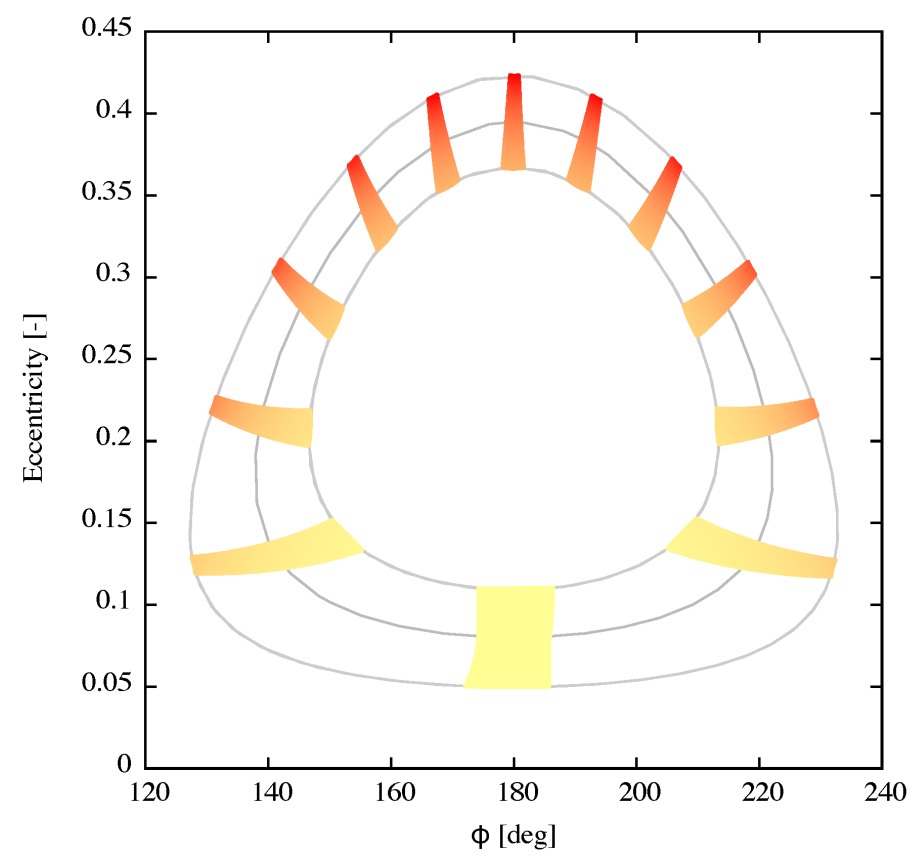

Fig. 3 Phase space evolution of initial set with uniform density over one loop period.

riod, the difference between the minimum and maximum density decreases again and approaches almost the initial density.

The same effect is visible also over several turns. In Figure 4(b) the evolution over three complete periods is shown. The periodic expansion and compression of the density and in particular the non-uniform density evolution within the initial set during a single period is clearly visible.

From the previous figures it appears as if the density is changing periodically. In case of only solar radiation pressure in this model, this is indeed the case as was shown by an analytical analysis of the Hamiltonian of that system as a consequence of Liouville's theorem (Colombo and McInnes, 2011). In case of the presence of $J_{2}$, however, this is not the case, as a more careful long term analysis shows. In Figure 4(c) we show the minimum and maximum density after each completed period for 15 periods. In this representation, which is ignoring the intermediate pulsating density evolution during each period, it becomes apparent that the minimum and maximum density in each turn is indeed drifting apart with time.

In Figure 6 the reason for this evolution becomes clear. As the initial set evolves, different parts move at different speeds resulting in a more and more non-linear shape and less uniform density distribution. Note that even in this case a single polynomial expansion is sufficient to accurately represent both the shape in phase space as well as the density of the cloud. This demonstrates the power of the DA density propagation method even under long term evaluation. 


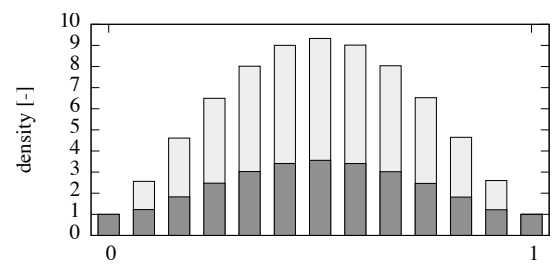

period [-]

(a) Over one period

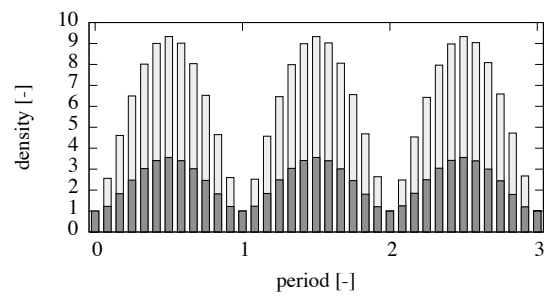

(b) Over three periods

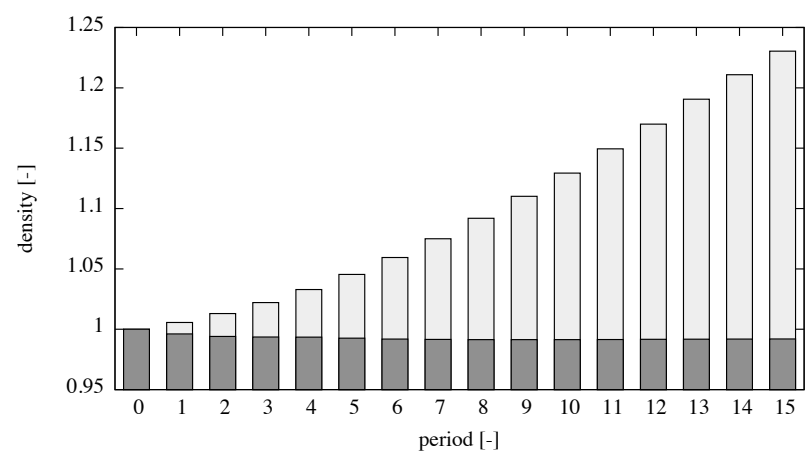

(c) Over 15 periods shown at each period

Fig. 4 Evolution of the minimum and maximum density.

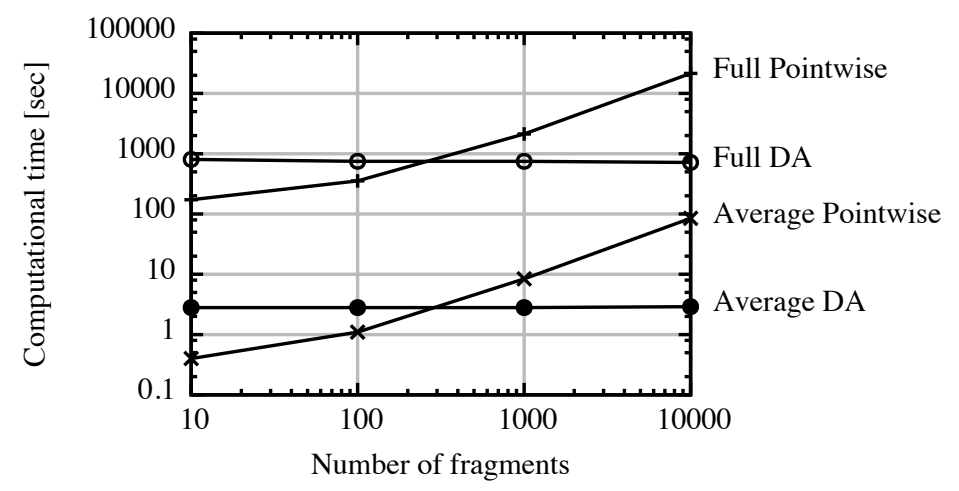

Fig. 5 Comparison of the computational speed for full and averaged dynamics with both DA and pointwise propagation as a function of the number of fragments. 


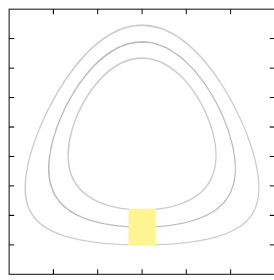

(a) initial set

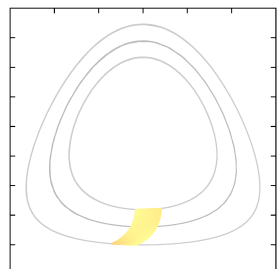

(e) period 4

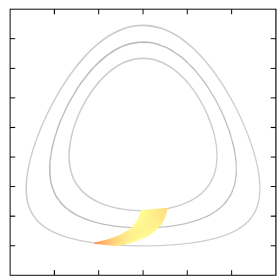

(i) period 8

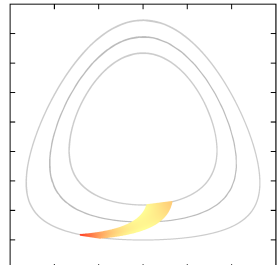

(m) period 12

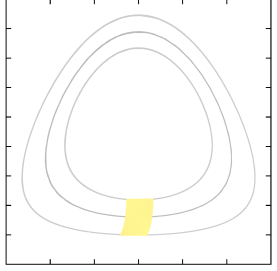

(b) period 1

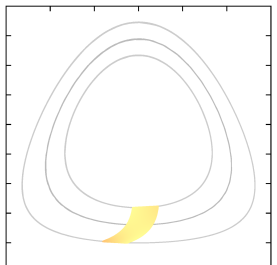

(f) period 5

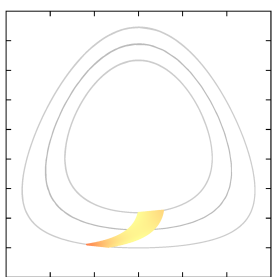

(j) period 9

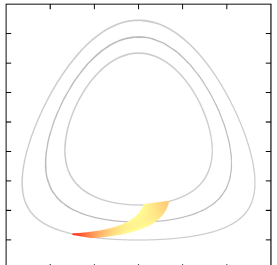

(n) period 13

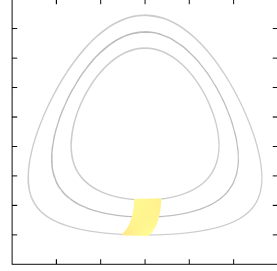

(c) period 2

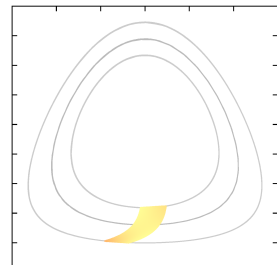

(g) period 6

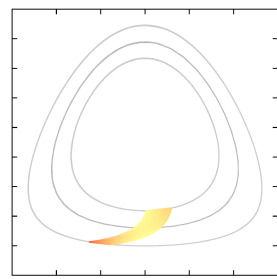

(k) period 10

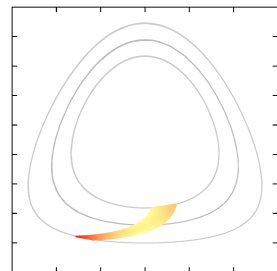

(o) period 14

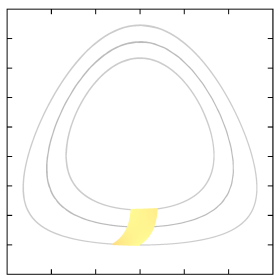

(d) period 3

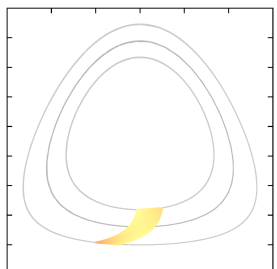

(h) period 7

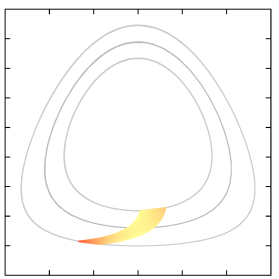

(l) period 11

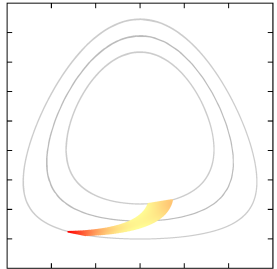

(p) period 15

Fig. 6 Long term evolution of initial set with uniform density distribution after every period. 
order of tens of seconds on an ordinary iMac with an $2.9 \mathrm{GHz}$ Intel Core i5 processor and 8 GB DDR3 RAM.

More precisely, Figure 5 shows the computation time required to propagate a given number of initial conditions and their associated densities for 1 period, corresponding to Figure 3. Two different dynamics, the full system and the averaged equations, are shown. Each dynamic is then integrated once with classical pointwise integration and once with DA integration. As expected, the computational cost of the averaged equations, both pointwise and DA, is lower than the computational cost of the full system by about two orders of magnitude. Furthermore, for the pointwise propagation it is clear that the cost of propagating $N$ points grows linearly with $N$. This is where the key advantage of DA propagation comes in: the cost of a DA propagation is essentially constant. This is because the propagation of a fragment only requires a single polynomial evaluation, the cost of which is negligible compared to the integration. Therefore, the DA propagation shows a large initial cost of computing the polynomial expansion of the flow once, about two orders of magnitude higher than the cost of a single pointwise propagation. However, once the polynomial is computed there is practically no additional cost for the propagation of virtually any number of fragments. Thus the break-even point is reached at only about 200-300 fragments, after which the initial cost of computing the polynomial in the DA propagation method is recovered and it becomes more efficient than pointwise propagation.

As mentioned before, the same method allows the propagation of arbitrary initial density distributions without the need to redo the integration. To illustrate, Figures 7(a) and 7(b) show the evolution of a non-uniform initial density distribution. In the first figure, a single Gaussian density distribution in both $\phi$ and $e$ is placed in the center of the initial condition box (centered at $\phi=180 \operatorname{deg}$ and $e=0.08$ with $\sigma_{\phi}=1.9 \mathrm{deg}$ and $\sigma_{e}=0.01$ ). In the second figure, two initially identical Gaussians are placed each $1 / 8$ away from the top and bottom respectively of the initial condition box (centered at $\phi=180$ deg and $e=0.0575$ and $e=0.1025$, respectively, with $\sigma_{\phi}=2.85 \mathrm{deg}$ and $\left.\sigma_{e}=0.015\right)$. As can be seen in Figure 7(b), the density of the two Gaussians evolves differently: the density of the outer Gaussian increases significantly more than the density of the inner Gaussian.

While all these results are qualitatively correct and match expectations, we also performed a quantitative accuracy analysis. To that end, a grid of 50 sample points was chosen in the initial box. The initial conditions and densities corresponding to those points are then propagated forward using the DA density propagation method as well as the continuity equation method developed by the authors in earlier works (Colombo and McInnes, 2011). For the purpose of this comparison, the pointwise integration of the continuity equation is considered to be the correct value. Propagation is performed for one full period, while taking snapshots at the same equally spaced time intervals shown in Figure 3.

At each point of the grid the error between the DA density $p_{D A}$ and the density $p_{C E}$ from the continuity equation is computed. The maximum error over all time slices at each point is shown in Figure 8. The white cross in the center of the box marks the expansion point. As can be seen from the 


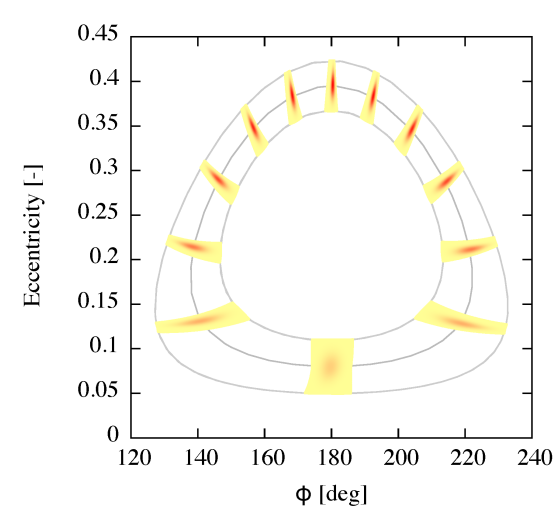

(a) Gaussian density distribution

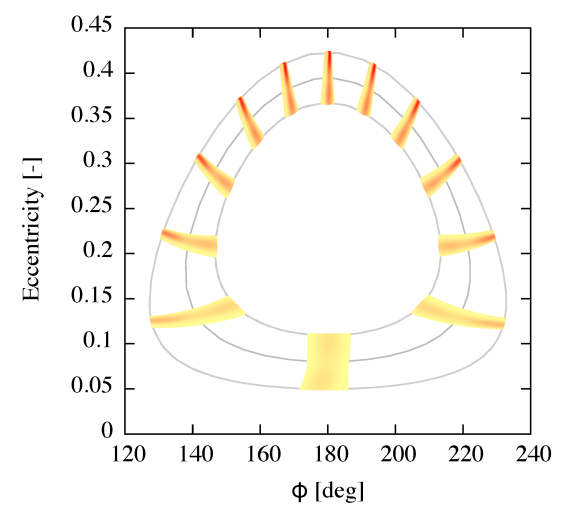

(b) Mixture of two Gaussian density distributions

Fig. 7 Evolution of non-uniform initial density distributions.

different scales on the color bars in each plot, with increasing computation order also the accuracy of the method improves significantly. Furthermore, convergence in $\Phi$ is quickly achieved, so that already at order 6 the relative error only increases with eccentricity $e$ but remains constant over all values of $\Phi$.

Figure 9 further illustrates the dependence of the maximum error of the DA density propagation on the computation order. The error shown is the maximum error over all points in the grid and all times. As is expected of a Taylor expansion, the error decreases exponentially up to about order 8 . However, for orders 9 and 10 the error does not decrease any more significantly. This is likely due to floating point errors accumulated in the computation of the high order coefficients. This effect is more pronounced in the higher order terms, because of the higher number of contributions to these terms during their computation as well as their small size. Practically, this limits the influence of the computation order at double precision. To lower the error further, instead of increasing the order further it is more efficient to subdivide the initial condition box and cover it by several smaller Taylor expansions using an automated domain splitting technique (Wittig et al., 2015).

\section{$6.2 J_{2}, \mathrm{SRP}$ and full luni-solar Dynamics}

Once the phase space evolution is clear we can now replace the simplified model of the dynamics with a more accurate model including perturbations due to $J_{2}$, SRP and the luni-solar gravitational potential using real ephemeris data for the Sun and Moon. The obliquity angle of the ecliptic over the equator is set to $\varepsilon=23.4393 \mathrm{deg}$ and the real ephemerides of the Earth around the Sun as well as the Moon around the Earth are considered, based on a low order polynomial analytical model. 


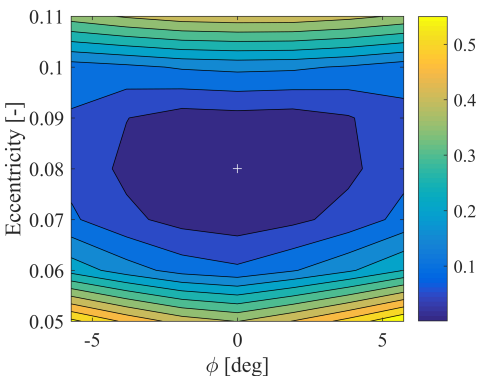

(a) Order 2

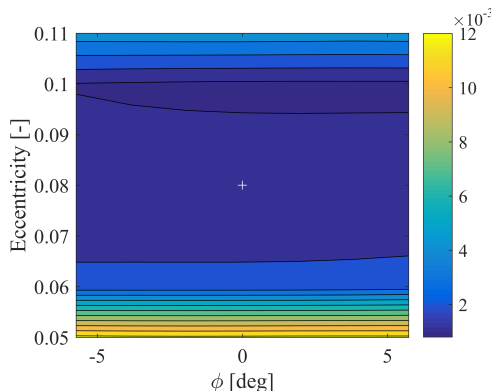

(c) Order 6

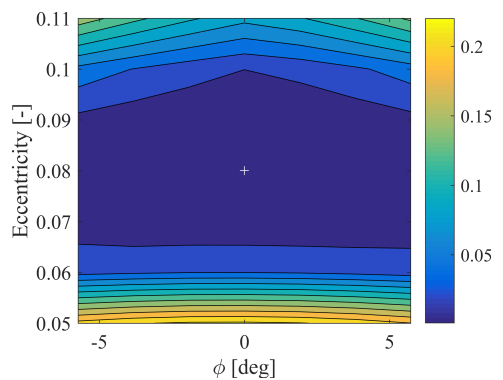

(b) Order 3

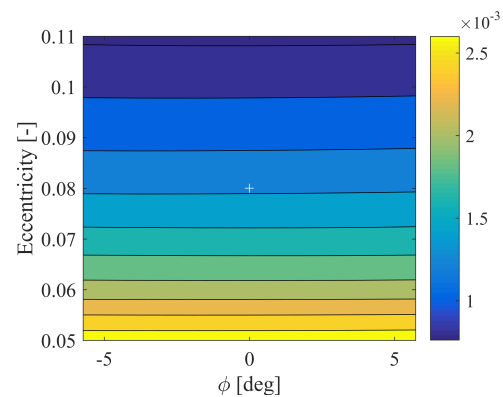

(d) Order 10

Fig. 8 Absolute error of DA density propagation with respect to pointwise integration of the continuity equation at different computation orders.

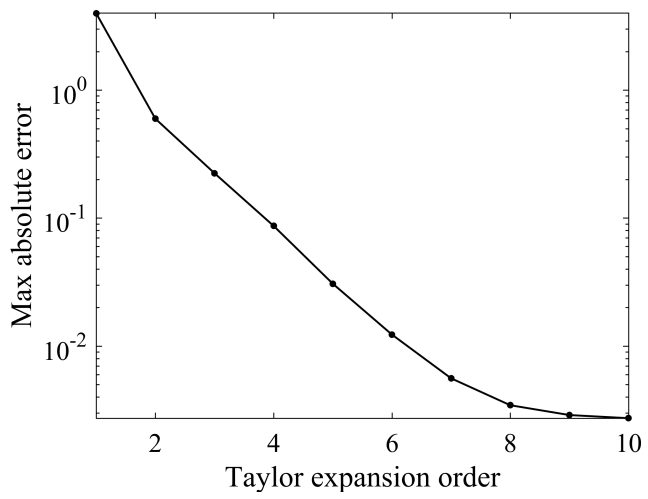

Fig. 9 Maximum absolute error of DA density propagation for different computation orders.

As Figure 10 shows, including luni-solar perturbations and a more realistic ephemeris of the Sun leads to non-periodic motion. However, the DA method for density propagation is working the same way as before without changes to the code after changing the dynamical model. The additional terms in the dynamical model do not require any further work for the density propagation. 


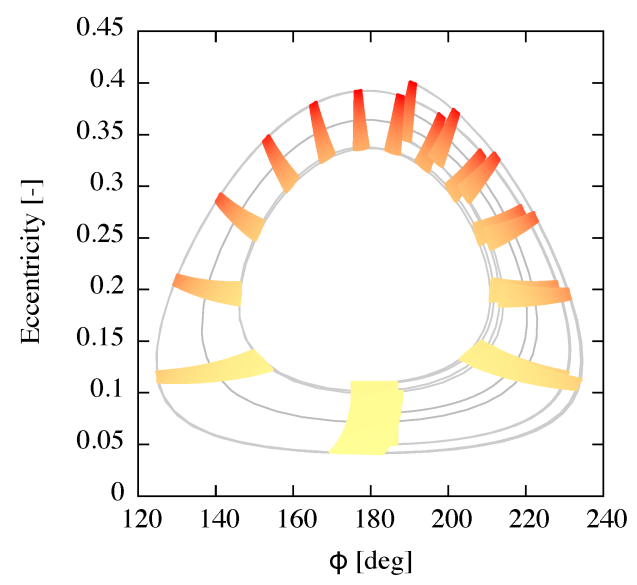

Fig. 10 Phase space evolution of initial set with uniform density over one period.

\section{Conclusions}

In this paper, we introduced a DA based method for the propagation of sets of initial conditions in phase space and their associated phase space densities, and then applied this technique to a DA enabled implementation of SA dynamics.

The DA based method for the propagation of densities introduced in this paper is independent of the dynamical system, and can be applied to both averaged (SA) as well as non-averaged (high fidelity) dynamics. However, the particular combination of DA techniques with SA equations yields a fast and accurate method for propagation of large clouds of initial conditions and their associated probability density functions very efficiently for long time.

The evolution of a cloud of high area-to-mass objects in MEO is reproduced considering the effects of solar radiation pressure, the Earth's oblateness and luni-solar perturbation. The computational efficiency is demonstrated by propagating 10,000 random samples taking snapshots of the position and density at each point throughout the integration. The time required for an integration of 16 years is on the order of seconds on an ordinary desktop PC. This is several orders of magnitude below the computational time consumed by the integration of the continuity equation for the same points.

As a future work, coupling the expression of the density in the phase space with the density over one single orbit (derived under a two-body approximation), will allow recovering the full $6 \mathrm{D}$ density of high area-to-mass objects lost due to the averaging over the fast variable $M$. This opens the door to applications in the description of debris evolution.

Moreover, the coupling or DA mapping with SA techniques will be investigated as an alternative to double averaged techniques and DA domain splitting will be employed in some case where the dynamics exhibit strongly non-linear behavior in the chaotic regime. 
Acknowledgments

A. Wittig gratefully acknowledges the support received from the Marie Curie network PITN-GA 2011-289240 (AstroNet-II); he performed part of this work at the Department of Aerospace Science and Technology, Politecnico di Milano, Italy. C. Colombo acknowledges the support received by the Marie Curie Intra European Fellowship (SpaceDebECM - Space Debris Evolution, Collision risk, and Mitigation - IEF-2011-302270) within the $7^{\text {th }}$ EU Framework Programme.

\section{References}

Armellin, R., Di Lizia, P., Bernelli Zazzera, F., Berz, M.: Asteroid close encounter characterization using differential algebra: the case of aphophis. Celestial Mechanics and Dynamical Astronomy 107(4) (2010)

Battin, R.: An Introduction to the Mathematics and Methods of Astrodynamics. AIAA Education Series, Reston, VA (1999)

Berz, M.: The method of power series tracking for the mathematical description of beam dynamics. Nuclear Instruments and Methods A258 (1987)

Berz, M.: Modern Map Methods in Particle Beam Physics. Academic Press (1999)

Berz, M., Makino, K.: COSY INFINITY version 9 reference manual. Michigan State University, East Lansing, MI 48824 (2006). MSU Report MSUHEP060803

Broucke, R.A.: Long-term third-body effects via double averaging. Journal of Guidance, Control, and Dynamics 26(1) (2003). DOI 10.2514/2.5041

Colombo, C.: Long-term evolution of highly-elliptical orbits: luni-solar perturbation effects for stability and re-entry. In: AAS/AIAA Space Flight Mechanics Meeting, Williamsburg, Virginia, AAS-15-395 (2015)

Colombo, C., Lücking, C., McInnes, C.: Orbital dynamics of high area-tomass ratio spacecraft with $J_{2}$ and solar radiation pressure for novel earth observation and communication services. Acta Astronautica 81(1), 137150 (2012). DOI 10.1016/j.actaastro.2012.07.009

Colombo, C., McInnes, C.R.: Evolution of swarms of smart dust spacecraft. In: New Trends in Astrodynamics and Applications VI, New York, June 2011 (2011)

Deprit, A.: Canonical transformations depending on a small parameter. Celestial mechanics 1(1), 12-30 (1969). DOI 10.1007/BF01230629

G., M., P., E.: Semi-analytical theory of the mean orbital motion. Astronomy and Astrophysics 294, 278-286 (1994)

Gor'kavyi, N.: A new approach to dynamical evolution of interplanetary dust. The Astrophysical Journal 474(1), 496-502 (1997). DOI 10.1086/303440

Krivov, A.V., Sokolov, L.L., Dikarev, V.V.: Dynamics of mars-orbiting dust: Effects of light pressure and planetary oblateness. Celestial Mechanics and Dynamical Astronomy 63(3), 313-339 (1995). DOI 10.1007/bf00692293

Letizia, F., Colombo, C., Lewis, H.G.: Multidimensional extension of the continuity equation method for debris clouds evolution. Advances in Space Research 57(8), 1624-1640 (2015). DOI 10.1016/j.asr.2015.11.035 
McInnes, C.R.: An analytical model for the catastrophic production of orbital debris. ESA Journal 17(4), 293-305 (1993)

Nazarenko, A.I.: Collision of spacecraft with debris particles assessment. 17th ISSFD Symposium. Moscow, Russia (2003)

Postnikov, M.M.: Geometry VI: Riemannian Geometry, Encyclopaedia of Mathematical Sciences, vol. 91. Springer (2001)

Rossi, A., Anselmo, L., Cordelli, A., Farinella, P., Pardini, C.: Modelling the evolution of the space debris population. Planetary and Space Science 46(11-12), 1583-1596 (1998). DOI 10.1016/S0032-0633(98)00070-1

Soong, T.: Fundamentals of Probability and Statistics for Engineers. Wiley (2004)

Valli, M., Armellin, R., Di Lizia, P., Lavagna, M.R.: Nonlinear mapping of uncertainties in celestial mechanics. Journal of Guidance, Control, and Dynamics 36(1), 48-63 (2013). DOI 10.2514/1.58068

Wittig, A., Armellin, R., Colombo, C., Di Lizia, P.: Long-term orbital propagation through differential algebra transfer maps and averaging semianalytical approaches. In: AAS/AIAA Space Flight Mechanics Meeting, Santa Fe, NM, AAS 14-224 (2014)

Wittig, A., Di Lizia, P., Armellin, R., Makino, K., Bernelli Zazzera, F., Berz, M.: Propagation of large uncertainty sets in orbital dynamics by automatic domain splitting. Celestial Mechanics and Dynamical Astronomy 122(3), 239-261 (2015). DOI 10.1007/s10569-015-9618-3 


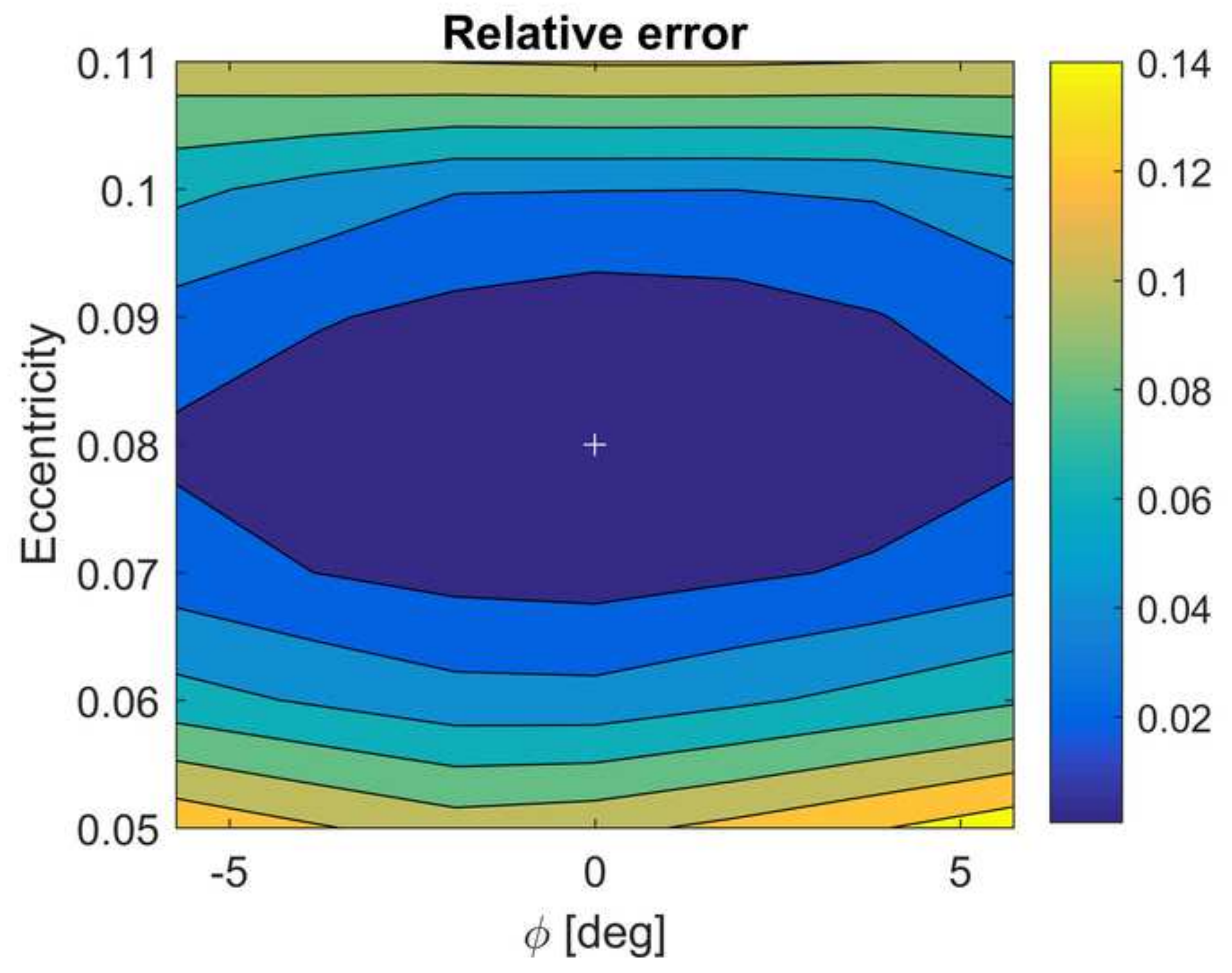




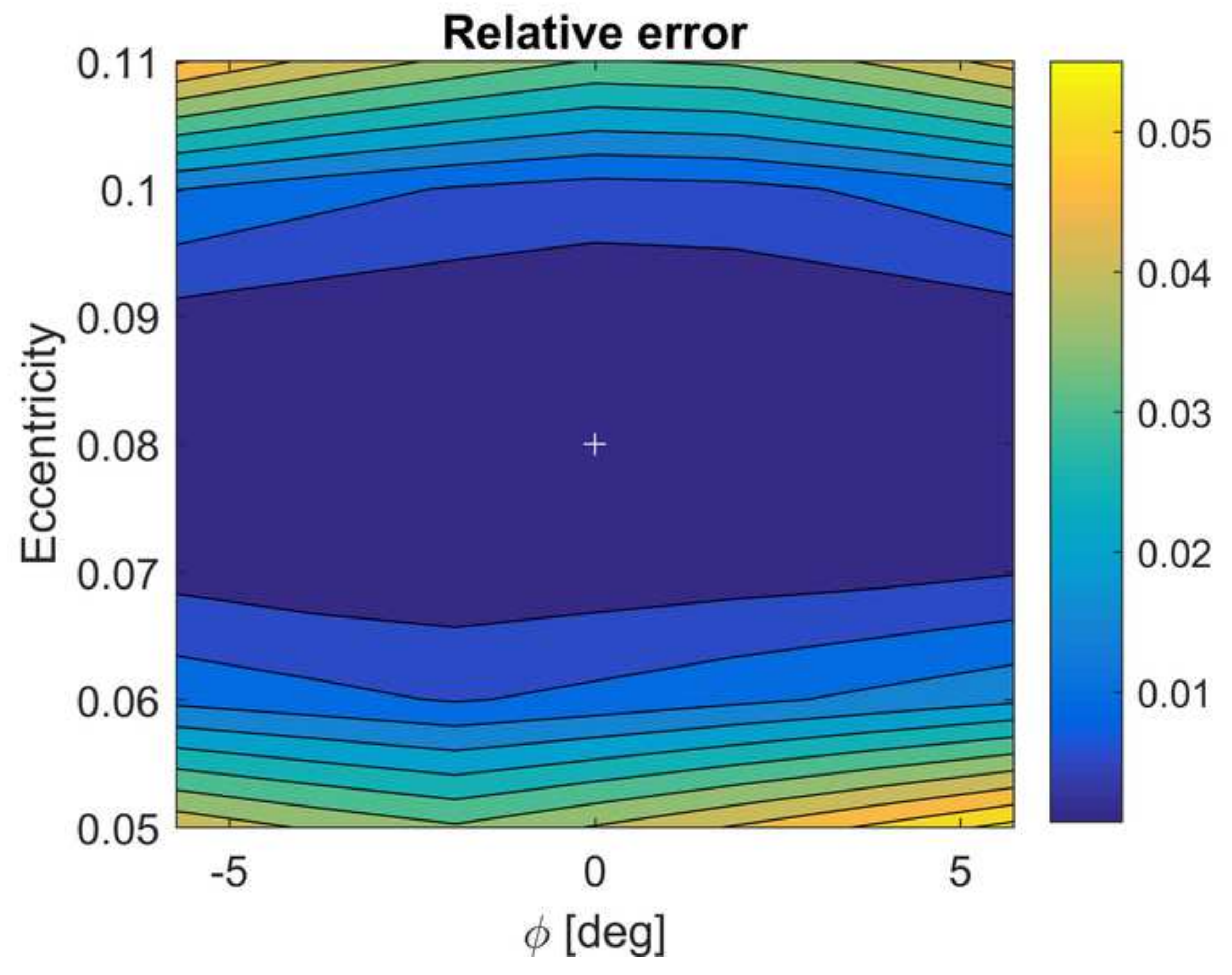




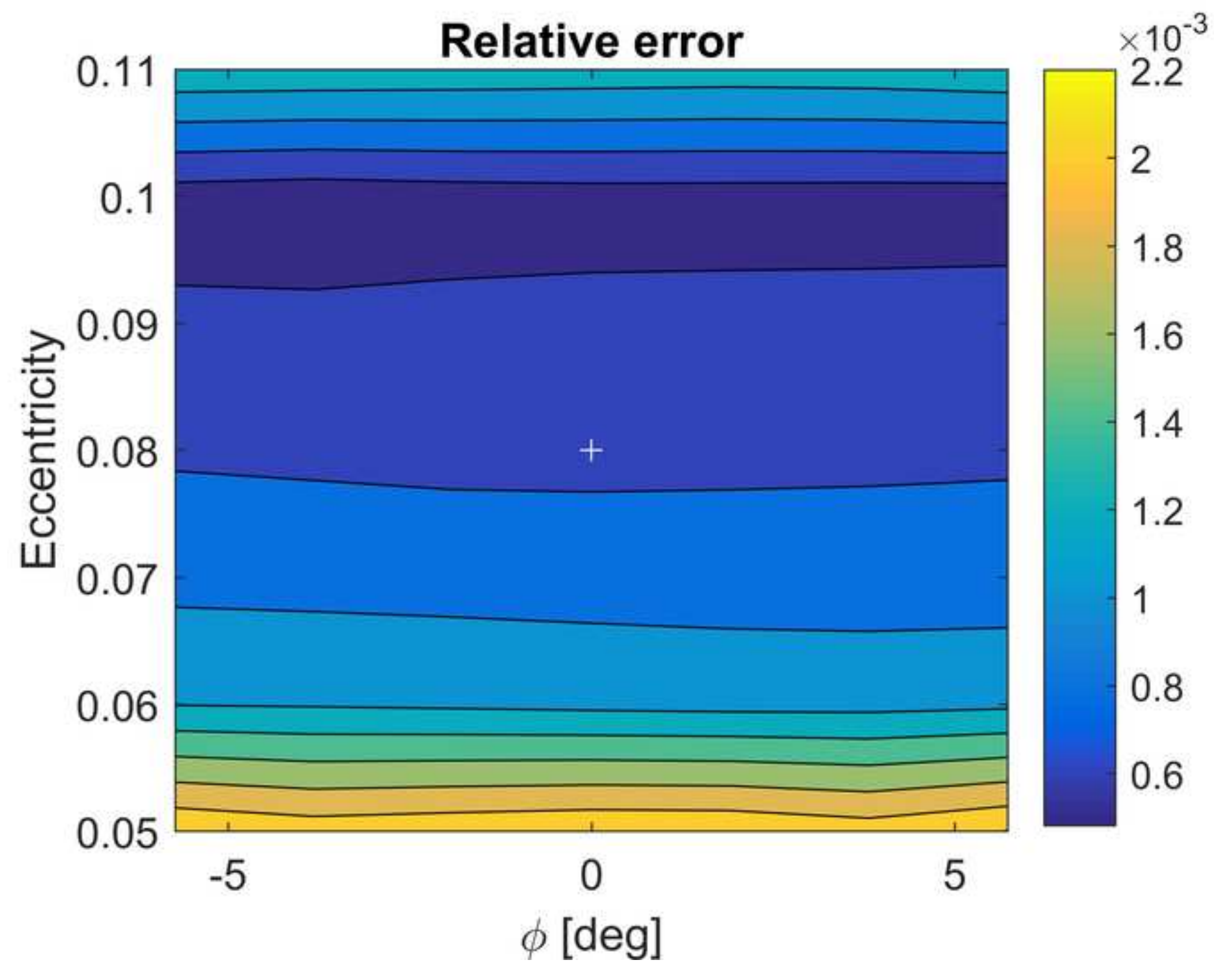




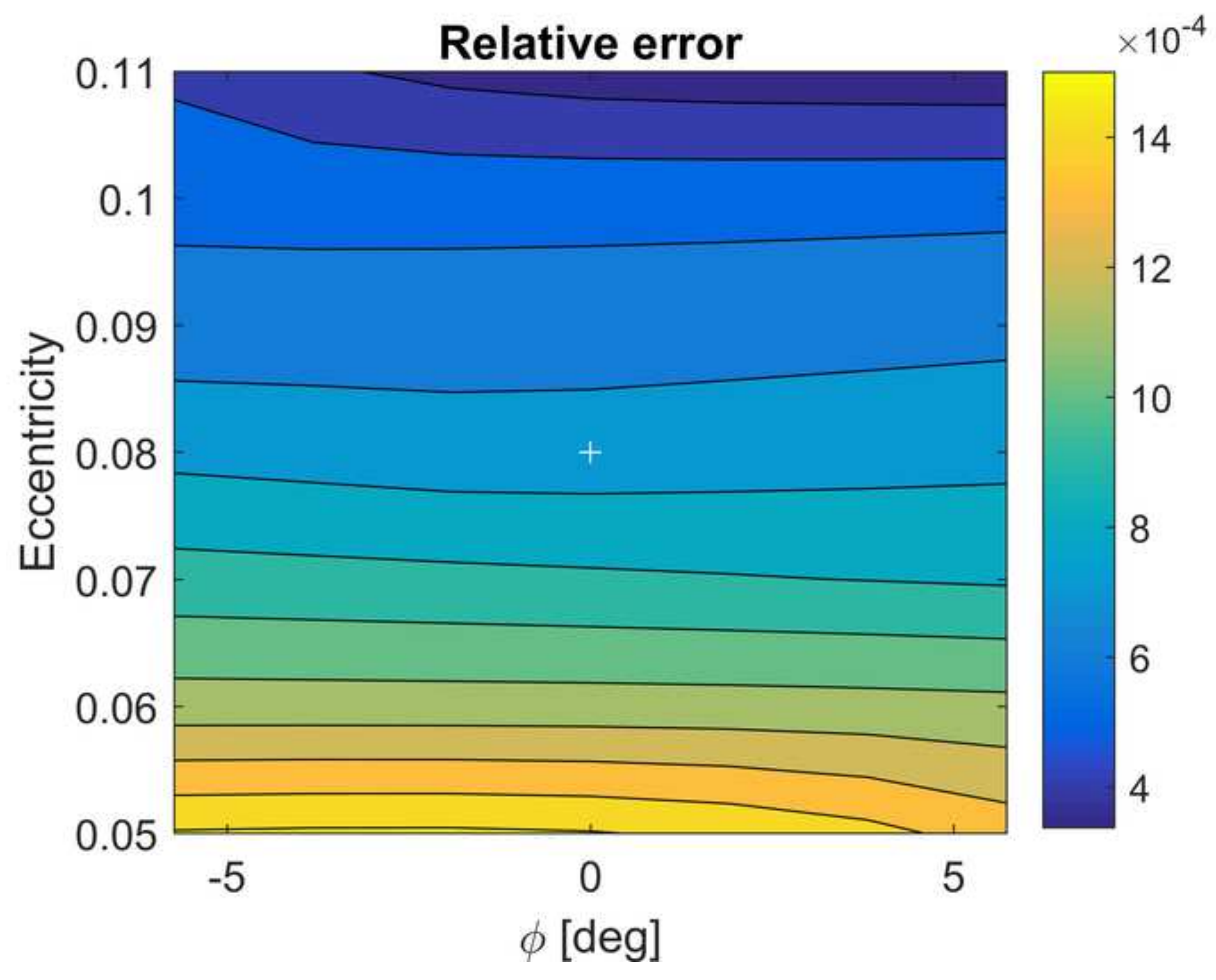




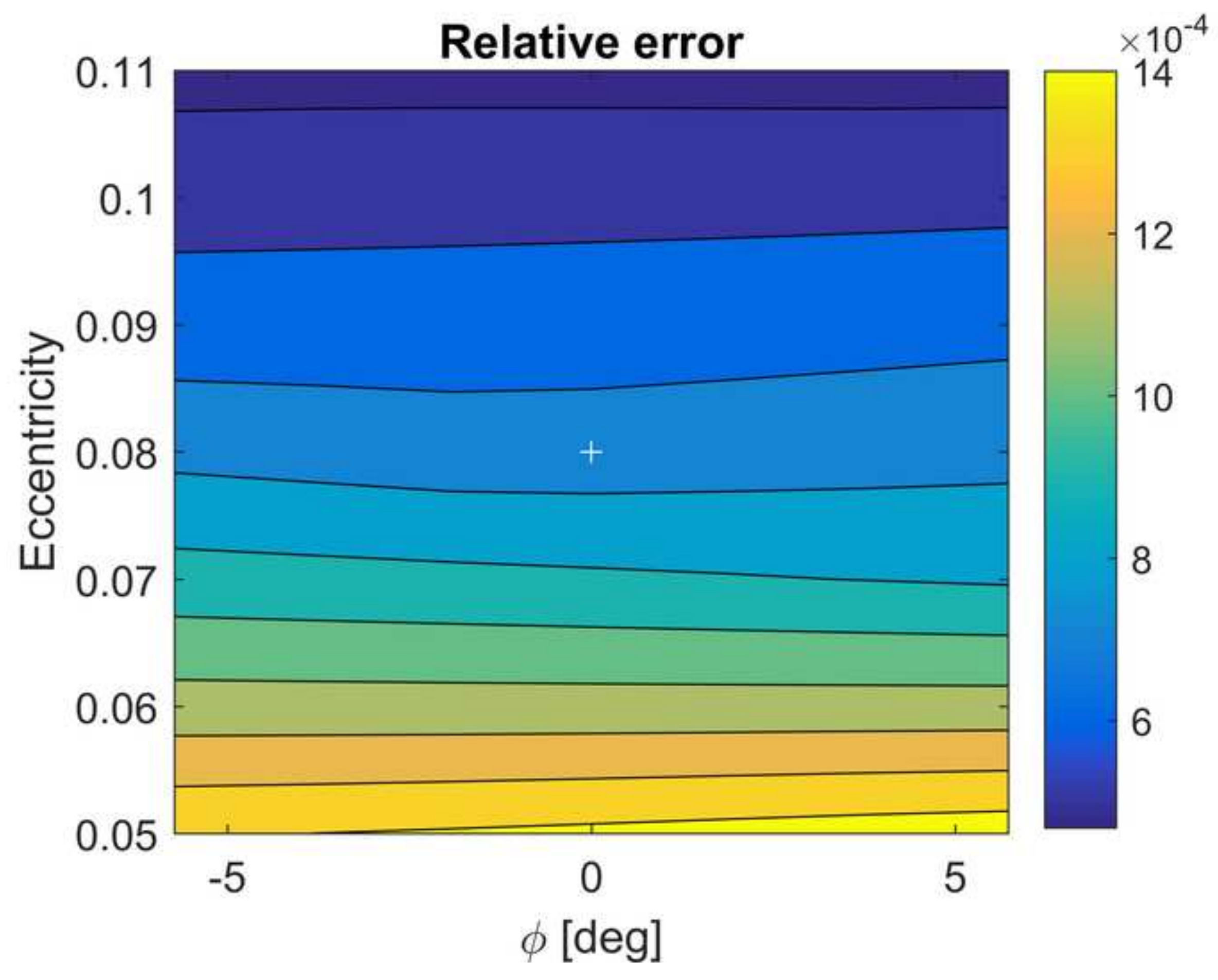


Click here to download Figure case7-1-errRelOr.png $\stackrel{ \pm}{ }$

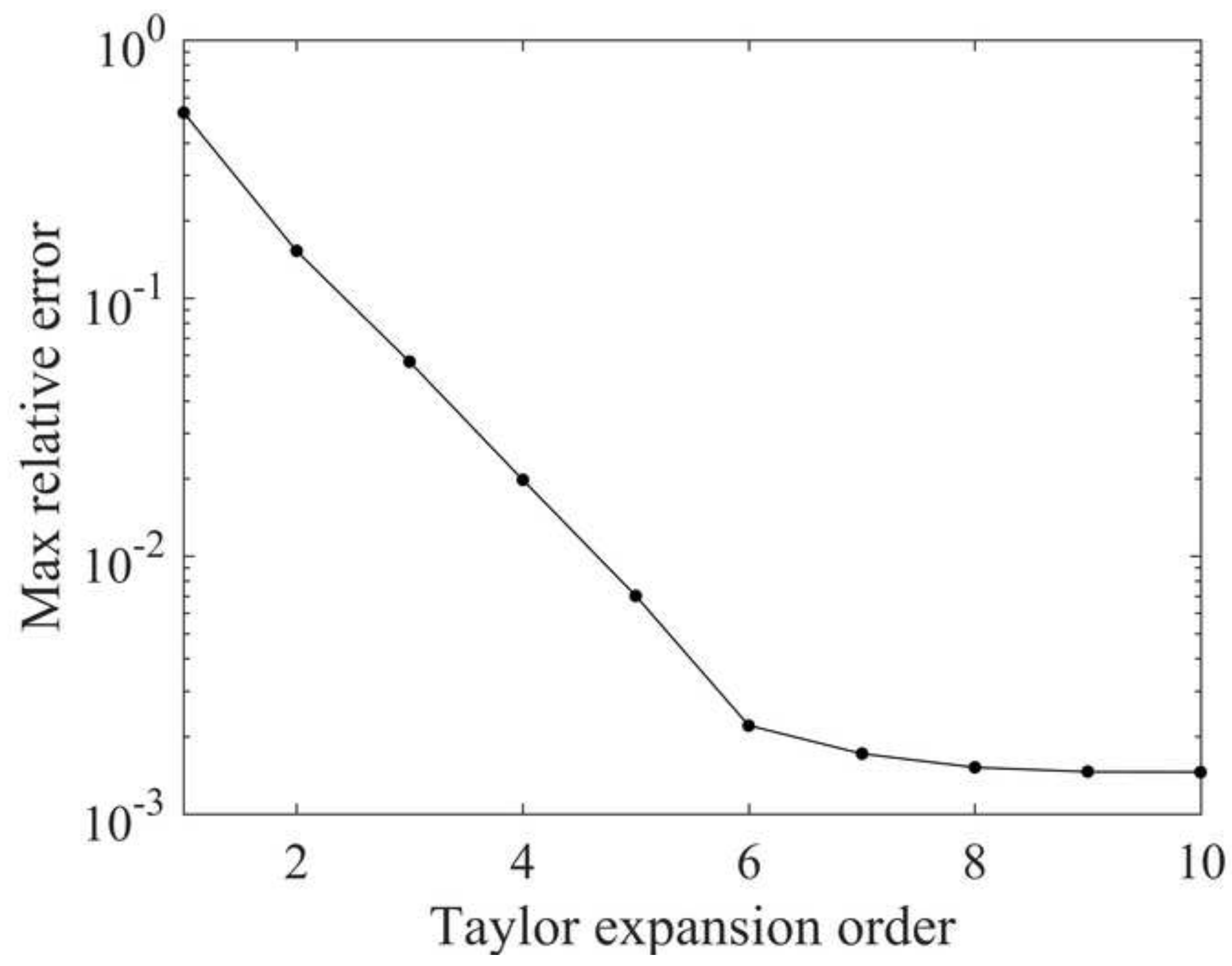




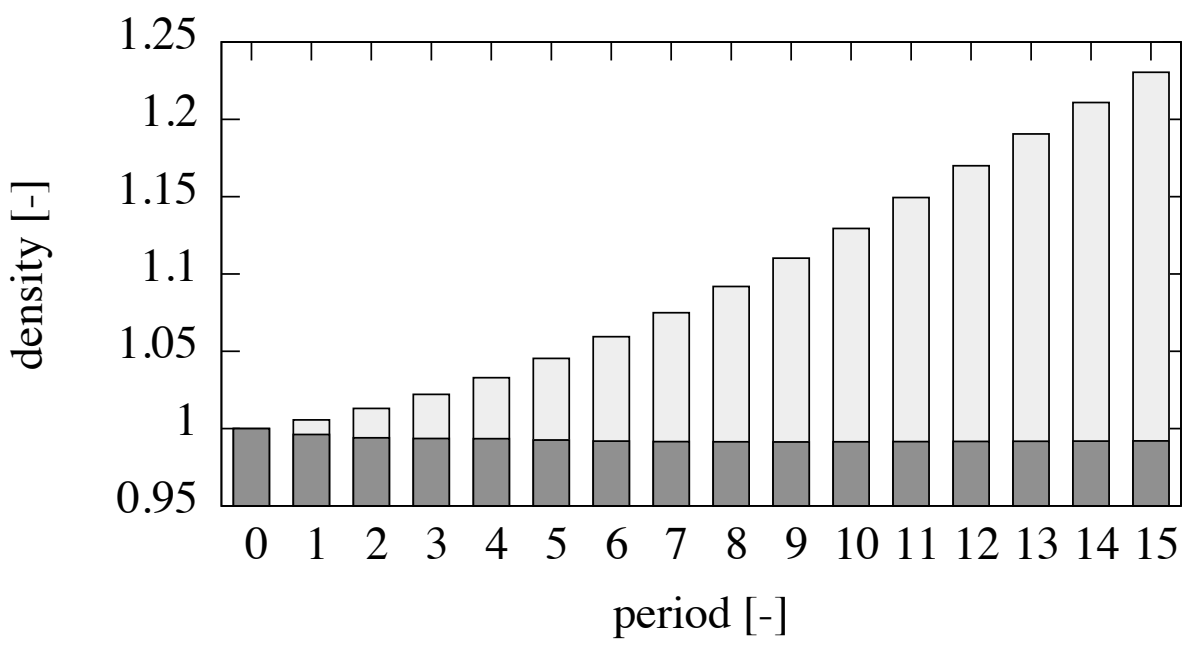

